\title{
Comparing canopy density measurement from UAV and hemispherical photography: an evaluation for medium resolution of remote sensing-based mapping
}

\author{
Deha Agus Umarhadi ${ }^{1}$, Projo Danoedoro ${ }^{2}$ \\ ${ }^{1,2}$ Remote Sensing Laboratory, Faculty of Geography, Universitas Gadjah Mada, Indonesia \\ ${ }^{1}$ Graduate School of Environmental Science, Faculty of Environmental Earth Science, Hokkaido University, Japan
}

\begin{abstract}
Article Info
Article history:

Received Mar 30, 2020

Revised Jun 14, 2020

Accepted Jun 27, 2020

Keywords:

Canopy density

Hemispherical photography

Image processing

Landsat-8

Remote sensing

UAV

Vegetation index

ABSTRACT

UAV and hemispherical photography are common methods used in canopy density measurement. These two methods have opposite viewing angles where hemispherical photography measures canopy density upwardly, while UAV captures images downwardly. This study aims to analyze and compare both methods to be used as the input data for canopy density estimation when linked with a lower spatial resolution of remote sensing data i.e. Landsat image. We correlated the field data of canopy density with vegetation indices (NDVI, MSAVI, and AFRI) from Landsat-8. The canopy density values measured from UAV and hemispherical photography displayed a strong relationship with 0.706 coefficient of correlation. Further results showed that both measurements can be used in canopy density estimation using satellite imagery based on their high correlations with Landsat-based vegetation indices. The highest correlation from downward and upward measurement appeared when linked with NDVI with a correlation of 0.962 and 0.652 , respectively. Downward measurement using UAV exhibited a higher relationship compared to hemispherical photography. The strong correlation between UAV data and Landsat data is because both are captured from the vertical direction, and $30 \mathrm{~m}$ pixel of Landsat is a downscaled image of the aerial photograph. Moreover, field data collection can be easily conducted by deploying drone to cover inaccessible sample plots.
\end{abstract}

This is an open access article under the CC BY-SA license.

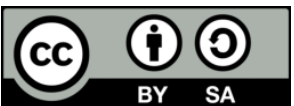

\section{Corresponding Author:}

Projo Danoedoro,

Remote Sensing Laboratory, Faculty of Geography,

Universitas Gadjah Mada,

Sekip Utara Bulaksumur Yogyakarta 55281, Indonesia.

Email: projo.danoedoro@geo.ugm.ac.id

\section{INTRODUCTION}

Information on vegetation canopy is essential for many applications such as hydrology, carbon and nutrient cycle, and global change study [1]. One example of the application is to evaluate the forest status and degradation which require canopy density data as the indicator for forestry management [2,3]. In addition, this biophysical information is also useful to infer the quality of the vegetation stands since the change in density indicates the degradation even though the extent remains the same $[2,4]$.

Canopy density estimation using remote sensing data is more efficient than traditional estimation due to the indirect measurement. Traditional estimation of canopy density requires extensive field surveys which take a long time and cover a relatively narrow area. There are various methods to estimate canopy density using remote sensing, such as empirical methods using regression, forest cover density mapper, and 
fractional vegetation cover [5-11]. However, the ground truth data of canopy density is needed to validate the estimation, also it is used by the empirical methods to build the model.

There are several photographic techniques for canopy description and the most widely used method is by utilizing hemispherical photography $[12,13]$. By using hemispherical photography, various aspects of vegetation can be obtained based on the geometry distribution of the gap fraction and it is beneficial to calculate forest light regimes and canopy properties, including canopy openness, leaf area index, and leaf angle distribution [14]. The upward-based photograph further eliminates the unnecessary vegetation properties, such as understory canopy information which is not captured by using this type of measurement [15].

The development of unmanned aerial vehicle (UAV) technology with small-format photographs as a cheap and efficient survey tool has triggered an application in vegetation monitoring. In the case of forestry and plantation inventory, UAV based mapping costs USD 3.58-4.78 per hectare which is much more affordable than field surveys [16]. Several studies implemented aerial photography to measure canopy density data based on the analysis of the objects [17], pixels [18], and point clouds [19]. Although the deployment is relatively cheap, to cover a larger area, medium resolution satellite imagery is more effective. Hence, the data derived from UAV is used to construct a canopy density model in medium resolution remote sensing imagery [20].

Hemispherical photography and aerial photography used in canopy density measurement represent contrast viewing angles which are upward and downward. It requires further assessment of its applicability when linked with remote sensing imagery. By comparing the UAV-based method and hemispherical photography then applying in $10 \mathrm{~m}$ resolution of the satellite image, Umarhadi et al. [21] found that the better canopy density estimation was provided by the upward method. This study used Landsat- 8 image which has a coarser resolution. The continuity and availability of Landsat data series provide the capability for national scale monitoring purposes [22]. This study aims to examine and compare hemispherical photography and aerial photography measurement as the input data in canopy density estimation using a medium resolution image of Landsat-8 OLI. Applied on the $30 \mathrm{~m}$ resolution, this study fulfilled the gap of the previous study which assessed $10 \mathrm{~m}$ of Sentinel-2 images.

\section{MATERIALS AND METHODS}

\subsection{Study area}

The study site is situated in Menoreh Mountains as shown in Figure 1, where lies in the western part of Special Region of Yogyakarta Province, as its border with Central Java Province, Indonesia. The mountainous morphology indicates the complexity of geomorphological processes that have been occurred. The minimum and maximum elevations are 12 and $988 \mathrm{~m}$ respectively, with slope ranges between $0^{\circ}-68^{\circ}$ according to SRTM data. Land use in this area is dominated by a mixed plantation which defined as the community forest. The vegetation types consist of woods (teak, mahogany, acacia), fruit (banana, jackfruit), crops (cassava, corn, soybean, peanut), and herbal plants (ginger, turmeric) [23]. This site was selected because it represents the typical community forest in Java Island, which needs to be monitored its sustainability to support the economy and the environment as well.

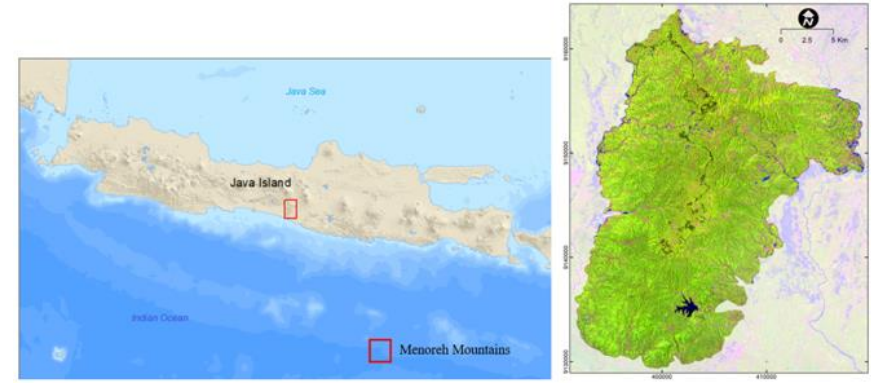

Figure 1. The location of Menoreh Mountains with the background of 654 image composite of Landsat-8

\subsection{Dataset}

Landsat-8 OLI imagery of 22 February 2015 was chosen for this study due to its cloud-free condition among other images. Landsat-8 OLI has $30 \mathrm{~m}$ spatial resolution and 16 days revisit time. Landsat- 8 has been widely used and it is reliable for vegetation studies in medium scale. The image was then pre-processed to correct the disturbance by mathematical models [24]. We obtained the Landsat data in the global geometric correction level, hence further geometric correction based on the local topographic map 
was conducted. The cirrus band in Landsat-8 OLI was used to minimize the haze disturbance and applied at the digital number (DN) level [25]. Radiometric TOA reflectance, as well as histogram adjustmentatmospheric correction, were applied after cirrus correction. Due to the mountainous topography, the topographic correction was also applied to the image by using Sun-Canopy-Sensor+C (SCS+C) method [26]. Afterward, we transformed the image into vegetation indices.

Several vegetation indices can be applied to the Landsat data, and each index has a unique characteristic. This study used three vegetation indices, NDVI, MSAVI, and AFRI. Landsat- 8 has two shortwaveinfrared so that AFRI consists of 2 indices, AFRI 1.6 and AFRI 2.1. The algorithms as shown in Table 1.

Table 1. Vegetation index algorithms

\begin{tabular}{ccc}
\hline Vegetation Index & Algorithm & References \\
\hline NDVI & $(\mathrm{rNIR}-\mathrm{rRed}) /(\mathrm{rNIR}+\mathrm{rRed})$ & {$[27]$} \\
MSAVI & $(\mathrm{rNIR}-\mathrm{rRed}) /(\mathrm{rNIR}+\mathrm{rRed}+\mathrm{L})) \times(1+\mathrm{L})$ & {$[28]$} \\
AFRI 1.6 & $(\mathrm{rNIR}-(0.66 \times \mathrm{r} \mathrm{r} \times \mathrm{N} .6)) /(\mathrm{rNIR}+(0.66 \times \mathrm{r} 1.6)$ & {$[29]$} \\
AFRI 2.1 & $(\mathrm{rNIR}-(0.5 \times \mathrm{r} 2.1)) /(\mathrm{rNIR}+(0.5 \times \mathrm{r} 2.1)$ & {$[29]$} \\
\hline
\end{tabular}

\subsection{Canopy density measurement}

Canopy density measurement was conducted by using two methods, hemispherical photography and aerial photography. DJI Phantom 3 Pro quad-copter was used for aerial photography measurement. Instead of using DJI's camera, color infrared (CIR) digital camera was mounted on the aerial platform for the measurement. This camera is the modification from Canon A2500. The measurement was conducted using Mission Planner software, then mosaicked, and cropped in $45 \times 45 \mathrm{~m}$, with the center coordinate is the same as the sample coordinate of Landsat- 8 image. We considered adding by $15 \mathrm{~m}$ to the $30 \times 30 \mathrm{~m}$ of Landsat- 8 resolution to accommodate the geometric movement probability according to McCoy [30]. Further analysis included the distinguishing of canopy and non-canopy object from the aerial photographs by using decision tree analysis.

Nikon DSLR camera with a fish-eye lens covering $180^{\circ}$ view was used for hemispherical photography measurement. Photos were captured from $170 \mathrm{~m}$ height of 5-7 photos upwardly. The captured area width was fixed to $45 \mathrm{~m}$ similar to downward measurement. Five until seven photos captured within $45 \times 45 \mathrm{~m}$, one photo in the center, and the others are in the surrounding. All of the photos were processed in Can-Eye software.

\subsubsection{Downward method}

The UAV flew seven times to take aerial photographs. The locations were scattered in the study area. Each deployment used a flight planner software as shown in Figure 2(a). Several points depicted on the flight plan are the sample plots that have planned before, and the line is the UAV flight path. The percentage of endlap and overlap was decided to accommodate the mosaic processing at the minimum number of $40 \%$. This mosaic was intended to reduce the effect of camera central projection and to generate orthophoto with orthogonal projection. Then, we processed georeferencing to obtain georeferenced photos. The orthophoto was sliced into several parts with the size of Landsat- 8 pixel which was added by geometry movement possibility, thus the sample area became $45 \times 45 \mathrm{~m}$. Some of the sample plots that had planned were changed because of the effect of central projection on the edge of photo mosaic. Figure 2(b) and 2(c) show one of the photo mosaics and some sample plots for the processing.

The camera used in this study, CIR camera, comprises blue, green, and near-infrared channels [31]. The photos had a contrast value in vegetation objects and were able to be distinguished other objects. Although containing 3 bands separately, the portion of near-infrared band appeared in the other bands, and it led the vegetation to obtain higher value in all bands. The results, however, are different from the multispectral camera which has a single band in each sensor or lens.

The percentage of canopy on aerial photographs was calculated based on the multispectral classification by using decision tree method. The photomosaics were not calibrated from one to the others. It affected the inconsistency values of the same object in different images. Therefore, the rule for each tree in different mosaics was different and derived by taking ROI in each photo mosaic. The number of sample classes depends on land cover variation in the photo. Basically, the classes were divided into canopy and non-canopy, but due to the presence of non-canopy objects that appeared at the top of the canopy, such as the rooftop, the classification class was modified into 3 classes, canopy, non-canopy, and rooftop as ahown in Figure 3(a) and 3(b). Decision tree classification result is a tree which its stems mean the classification rule as shown in Figure 3(c). CIR camera can distinguish canopy among the other objects greatly. For instance, in the sample 4(a), it is proven by the rule has purity over $99 \%$ for each class. 


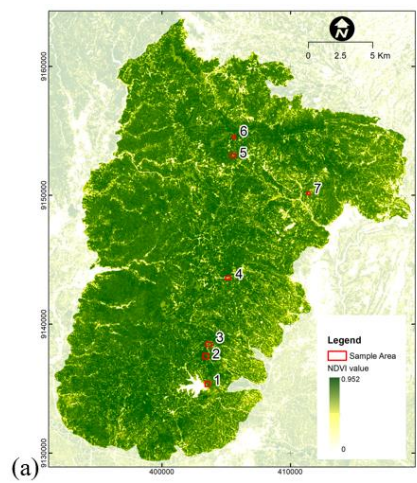

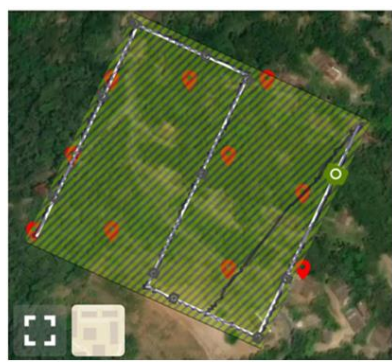

(b)

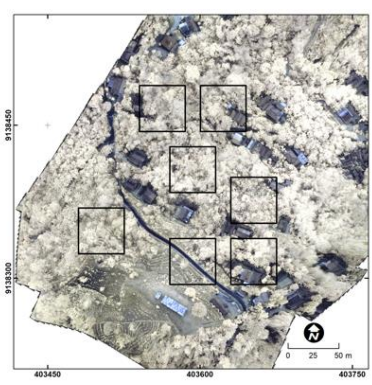

(c)

Figure 2. (a) The distribution of sample area locations with the background of NDVI image, (b) flight plan and (c) orthophoto mosaic in site 3

(a)
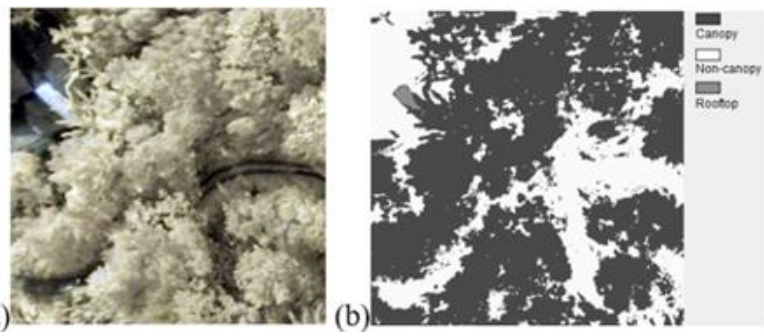

Roou

Band $3<179$

Band 1 र 156.5 Leat Class 2 [Pixels 1337: $88.22 \%$ ) Punly: 99.705 (c)
Band 1 > 156.5 Leat Class 1 [Pixels 1120: $95.96 \%$ ] Putity : $99.55 \%$

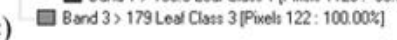

Figure 3. (a) Cropped image, (b) classification result, and (c) the rule of classification result of sample 4a

\subsubsection{Upward method}

Measurement and calculation of upward method were conducted by directing vertically upward using fish-eye lens camera and Can-Eye software, respectively. Before obtaining the photo, we did calibration first to examine the optical center, center of interest, and projection function, as the parameters of Can-Eye processing. The Center of interest used in this study is $60^{\circ}$, which means it does not calculate over $60^{\circ}$ of camera view due to the mixed pixel [32]. The processing procedure consists of selection, masking, and classification as shown in Figure 4. All photos which are located in the same sample spot were selected first, then the noise objects were cropped. The steep slope condition on some spots makes the vertically fish-eye camera captured the slope as shown in Figure 5. This condition also occurred in the site near the building and rooftop. As depicted in Figure 4, the building appeared on the photo. Slope and building are considered as noises and have been cropped on the processing.

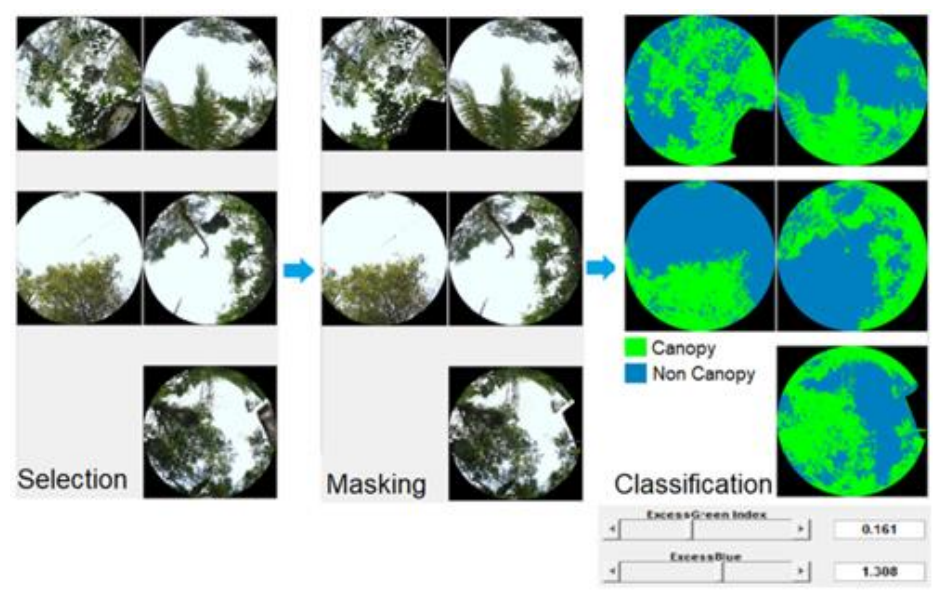

Figure 4. Upward classification process including selection, masking, and classification on sample 4a 

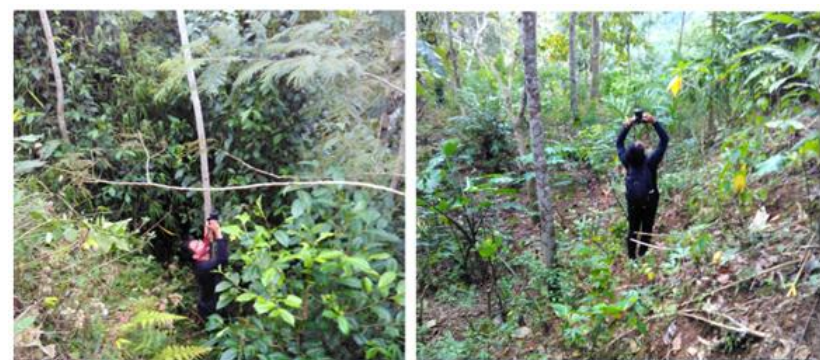

Figure 5. The field condition of upward measurement

Can-Eye software classification distinguished objects based on the threshold of 3 color indices, including ExcessGreen, ExcessBlue, dan ExcessRed. Since we did upward measurement, the classification used 2 indices consist of ExcessGreen and ExcessBlue. The percentage of green value over the area indicates the canopy density on the processing results.

\section{RESULTS AND DISCUSSION}

Correlation analysis was conducted to find out the intra-relationship between two methods by looking at the correlation of coefficient. Further, the correlation analysis between canopy density data and vegetation index value (NDVI, MSAVI, AFRI 1.6, and AFRI 2.1) was also conducted to measure the relationship level between the canopy density from the hemispherical and UAV photograph with the Landsat-8 data.

Both downward and upward measurement results were compared, with a total of 21 sample data as shown in Table 2. Both results have different canopy density values. There were 14 samples that had a different value of less than 10\%, 5 samples showed higher downward measurement results more than $10 \%$, and 2 samples showed higher upward measurement results of more than $10 \%$.

Table 2. Canopy density measurement results

\begin{tabular}{cccc}
\hline Sample Number & Upward & Downward & Downward - Upward \\
\hline 3f & 78.47 & 61.43 & -17.04 \\
3b & 69.17 & 54.81 & -14.36 \\
7b & 43.29 & 33.41 & -9.88 \\
4j & 38.04 & 31.45 & -6.59 \\
7d & 26.71 & 21.31 & -5.40 \\
4i & 50.23 & 45.54 & -4.69 \\
5e & 44.50 & 43.20 & -1.30 \\
5b & 72.98 & 73.21 & 0.23 \\
5a & 59.86 & 60.40 & 0.54 \\
2d & 70.71 & 73.44 & 2.73 \\
1p & 85.39 & 88.23 & 2.84 \\
4a & 63.51 & 66.51 & 3.00 \\
2c & 80.60 & 85.78 & 5.18 \\
5c & 72.60 & 79.13 & 6.53 \\
3d & 74.94 & 81.52 & 6.58 \\
5g & 74.18 & 82.13 & 7.95 \\
1b & 64.47 & 75.47 & 11.00 \\
1c & 49.91 & 70.23 & 20.32 \\
5f & 36.52 & 63.23 & 26.71 \\
6c & 39.36 & 69.50 & 30.14 \\
6b & 22.23 & 62.08 & 39.85 \\
\hline
\end{tabular}

Several factors led to the difference in the results. The first factor was the existence of understory canopy, where those objects were not captured in the upward measurement with the limit of captured height about $1.7 \mathrm{~m}$. On the other hand, downward measurement captured all objects including understory canopy. Unfortunately, the downward measurement not only captured understory canopy, but also shrub and herb. Shrub and herb were hardly differentiated from canopy by only using the spectral value. In some sample plots, shrub and herb were captured and they contributed to the overestimation of the measurement in downward measurement. 
The overestimate canopy density measurement due to the involvement of shrub and herb could be an advantage of the downward method for other applications such as erosion. However, the inclusion of shrub and herb more likely represented vegetation density, not canopy density. Erosion study considers basal area vegetation, such as dry leaves, grass, and other plant residues [33]. Downward canopy density measurement is more reliable to measure vegetation density, although it also can obtain canopy density, and it can be improved by masking the shrub and herb first.

The higher density in downward method was caused by the difficulty in classifying canopy object and soil background. Almost all soil backgrounds were covered by canopy layers and shadows, hence most of the tree crowns were classified as $100 \%$ of canopy. On the other hand, upward measurement was sensitive to distinguish canopy and sky background, as a result, there was no $100 \%$ density in each crown. The downward method term is closer to the definition of crown cover than canopy density [18]. Chianucci et al. [18] used true color (RGB) camera and mentioned that by using this method, and it was difficult to detect small within-crown gaps that were, much closer to crown cover, although it accurately quantified canopy cover. In this study, we used CIR camera. Although this camera includes a near-infrared band, it displayed the same problem to detect small gaps. This pixel-based classification may be improved by combining with point clouds derived canopy cover proposed by Khokthong et al. [19].

The hemispherical method has a different definition, according to Jennings [34], this method is used to measure canopy closure which means the proportion of the sky obscured by vegetation when viewed from a single point hemispherically. This definition is often used as the synonym of canopy density [34]. This term is different from canopy closure, which used ortho-vertical projection. The results between canopy closure and canopy cover are not necessarily correlated in the same area, because the height of the tree affects the amount of canopy proportion.

Although they had different results, both methods, downward and upward constructed a strong relationship. Correlation analysis showed that the 21 canopy density measurements from both methods showed a 0.71 correlation coefficient which means they had a strong relationship. On the scatterplot graph as shown in Figure 6, several samples showed that upward measurement value had a higher correlation of coefficient than downward measurement, but based on the normal line, more plots were placed in the proportion which indicates downward measurement results were higher than that of upward dominantly.

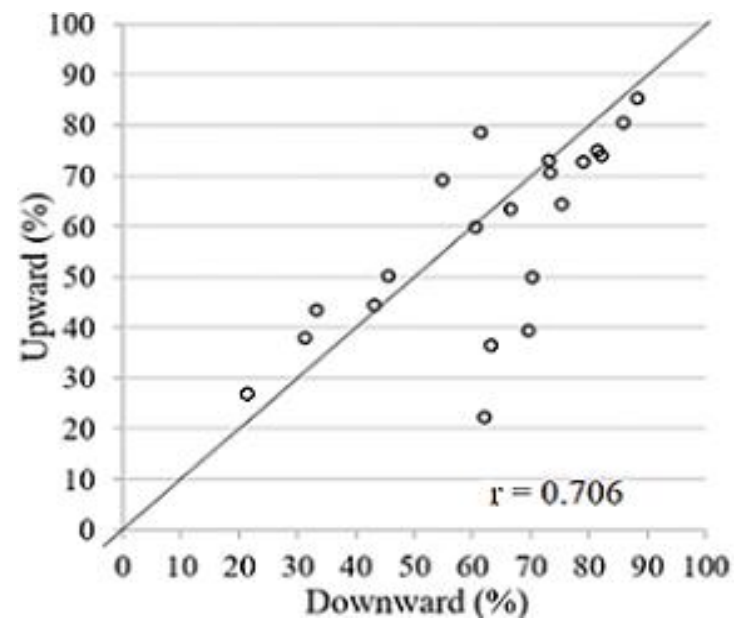

Figure 6. Plot of upward and downward measurement results

Canopy density estimation model in this study was constructed from vegetation index transformation, hence we also compared the correlation of both canopy density methods. Correlation analysis was applied in each method and each vegetation index. The correlation coefficients as shown in Table 3 showed the downward method possessed much stronger correlations on all of the vegetation indices compared to the upward method. The relationship of both methods with NDVI was the strongest among other vegetation indices, with the correlation in downward and upward measurements were 0.962 and 0.652 , respectively. This great correlation is mainly because NDVI has proven its capability in classifying land cover by the approach of vegetation density and estimating canopy density using remote sensing data [35]. We also included other vegetation indices, and all of them indicated that downward method performed better with higher correlation. 
Table 3. Correlation coefficients of vegetation indices and canopy density measurements

\begin{tabular}{ccc}
\hline Vegetation Index & \multicolumn{2}{c}{ Correlation Coefficient } \\
& Downward & Upward \\
\hline NDVI & 0.96153 & 0.65152 \\
MSAVI & 0.87973 & 0.54018 \\
AFRI 1.6 & 0.77952 & 0.59127 \\
AFRI 2.1 & 0.77617 & 0.56964 \\
\hline
\end{tabular}

The comparison to various vegetation indices yielded various levels of correlation. MSAVI and AFRI showed a different pattern in each correlation value of canopy density measurement. Correlation with MSAVI was higher than both methods against AFRIs in downward measurement, while it was conversely in upward measurement. The greater relationship between MSAVI and canopy data in downward method is because the algorithm of MSAVI considers the soil background effect. This measurement captured the whole objects from a height, thus soil background was included, and it was distinguished from canopy. Meanwhile, upward measurement did not consider soil background, and the consequence was the lower correlation with MSAVI.

The stronger relationship resulted in downward method was due to the objects captured in aerial photo depicted more detailed imagery similar to the Landsat-8 imagery when downscaled. This similar characteristic was not represented by using the upward method hence it produced a lower correlation. Several conditions affected the weaker correlation in upward method. The mountainous topography in the study area with a steep slope in some sample plots contributed to the improper measurement by using upward method, and the errors due to slopes were captured in the sample plot. The steep slope caused the removal of some areas in the sample plots. Moreover, it was difficult to apply this method in mountainous topography due to its inaccessibility.

The stronger relationship of downward measurement has been statistically proven by the comparison displayed in the scatterplot between both methods and NDVI value as shown in Figure 7. The scatterplot of downward measurement showed a linear relationship with $r=0.96$, and all plots lied close to the others. Meanwhile, the plots of upward measurement, in general, created a weaker linear relationship than downward, especially on the canopy density below $60 \%$.
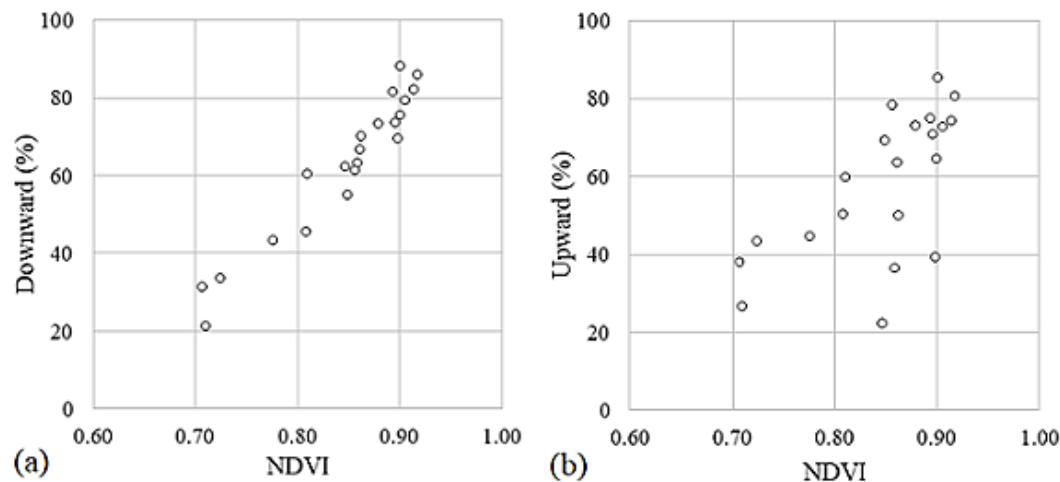

Figure 7. (a) Plot of downward and (b) upward measurement with NDVI value

\section{CONCLUSION}

Downward canopy density measurement using UAV has a different term with upward measurement using hemispherical photography, which has the closest definition to crown cover. Upward measurement using hemispherical photography is used to measure canopy cover, and this term is widely used to be the definition of canopy density. Although having a different definition, both measurements have a strong correlation.

Correlation between the measurements and vegetation indices showed that canopy density data obtained from downward and upward method can be used in canopy density estimation model using satellite imagery. UAV method has stronger correlations to vegetation indices of remote sensing data than another method because it depicts more detailed images of Landsat-8. Meanwhile, hemispherical photography is weaker due to the effect of topography and the presence of the building. The highest correlation was shown by using NDVI, with 0.962 and 0.652 correlation for downward and upward measurement. 
UAV imagery in this study showed similarity with the Landsat-8 OLI and resembled high relationships especially when downscaled into the resolution of the Landsat 8 . The similar information in both images resulted in a stronger correlation than the upward method. However, further adjustment by improving the detection of small gaps between canopies can be used to increase the prediction accuracy of using upward methods. Future study in improving the gaps detection in the upward method was deemed necessary to improve the relationship with the remote sensing imagery.

\section{ACKNOWLEDGEMENTS}

Authors would like to thank Sanjiwana Arjasakusuma for proofreading the paper. We also thank Ridho Dwi Dharmawan, Maulana Yudhinugroho, and Anisfa Andiyani for their helps in collecting field data.

\section{REFERENCES}

[1] C. Macfarlane, et al., "Estimation of Leaf Area Index in Eucalypt Forest Using Digital Photography," Agricultural and Forest Meteorology, vol. 143, no. 3-4, pp. 176-188, 2007.

[2] Z. Azizi, et al., "Forest Canopy Density Estimating, Using Satellite Images," The International Archives of the Photogrammetry, Remote Sensing and Spatial Information Sciences. vol. XXXVII, Part B8. Beijing, 2008.

[3] S. Arjasakusuma, et al., "Accuracy and Spatial Pattern Assessment of Forest Cover change Datasets in Central Kalimantan," Indonesian Journal of Geography, vol. 50, no. 2, pp. 222-227, 2018.

[4] N. R. Tohir, et al., "Pemetaan Perubahan Kerapatan Kanopi di Hutan Rakyat Kabupaten Kuningan Jawa BaratMapping of Canopy Density Change in Community Forest, Kuningan District, West Java (In Bahasa)," Seminar Nasional Penginderaan Jauh: Deteksi Parameter Geobiofisik dan Diseminasi Penginderaan Jauh, 322-331, 2014.

[5] F. Taureau, et al., "Mapping the Mangrove Forest Canopy Using Spectral Unmixing of Very High Spatial Resolution Satellite Images," Remote Sensing, vol. 11, no. 367, 2019.

[6] M. Kamal, et al., "Assessment of Mangrove Forest Degradation Through Canopy Fractional Cover in Karimunjawa Island, Central Java, Indonesia," Geoplanning: Journal of Geomatics and Planning, vol. 3, no. 2, pp. 107-116, 2016.

[7] S. Arjasakusuma, et al., "Local-Scale Accuracy Assessment of Vegetation Cover Change Maps Derived from Global Forest Change Data, ClasLite, and Supervised Classifications: Case Study at Part of Riau Province, Indonesia," Applied Geomatics, vol. 10, no. 3, pp. 205-217, 2018.

[8] C. Li, Mingyang Li, and Yingchang Li, "Improving Estimation of Forest Aboveground Biomass Using Landsat 8 Imagery by Incorporating Forest Crown Density as a Dummy Variable," Canadian Journal of Forest Research, vol. 50, no. 4, pp. 390-398, 2020.

[9] R. N. Khairiah, et al., "Agroforestry Tree Density Estimation Based on Hemispherical Photos \& Landsat 8 OLI/TIRS Image: a Case Study at Cidanau Watershed, Banten-Indonesia," The International Archives of the Photogrammetry, Remote Sensing and Spatial Information Sciences, vol. XLII-3/W7, 2019.

[10] J. C. Jiménez-Muñoz, et al., "Comparison Between Fractional Vegetation Cover Retrievals from Vegetation Indices and Spectral Mixture Analysis: Case Study of PROBA/CHRIS Data Over an Agricultural Area," Sensors, vol. 9, pp. 768-793, 2014.

[11] A. Abdollahnejad, P Dimitrios, S Peter., "Forest Canopy Density Assessment Using Different Approaches Review," Journal of Forest Science, vol. 63, pp. 106-115, 2017.

[12] F. Chianucci, U Chiavetta, A Cutini, "The Estimation of Canopy Attributes from Digital Cover Photography by Two Different Image Analysis Methods," iForest, vol. 7, pp. 255-259, 2014.

[13] F. Chianucci, "An Overview of In Situ Digital Canopy Photography in Forestry," Canadian Journal of Forest Research, vol. 50, no. 3, pp. 227-242, 2020.

[14] F. Chianucci and A. Cutini, "Digital Hemispherical Photography for Estimating Forest Canopy Properties: Current Controversies and Opportunities," iForest, vol. 5, pp. 290-295, 2012.

[15] F. Ashaari, M. Kamal, D. Dirgahayu, "Comparison of Model Accuracy in Tree Canopy Density Estimation Using Single Band, Vegetation Indices and Forest Canopy Density (FCD) Based on Landsat-8 Imagery (Case Study: Peat Swamp Forest in Riau Province)," Int. Journal of Remote Sensing and Earth Sciences, vol. 15, pp. 81-92, 2018.

[16] S. A. Suab and R. Avtar, "Unmanned Aerial Vehicle System (UAVS) Applications in Forestry and Plantation Operations: Experiences in Sabah and Sarawak, Malaysian Borneo," Unmanned Aerial Vehicle: Application in Environment and Agriculture, R. Avtar and T. Watanabe (Eds.), Cham: Springer International Publishing, 2020.

[17] Y. Erfanifard and Z. Khodaee, "Canopy Density Mapping on Ultracam-D Aerial Imagery in Zagros Woodlands, Iran," International Archives of the Photogrammetry, Remote Sensing and Spatial Information Sciences, vol. XL-1/W3, 2013 SMPR 2013, 2013.

[18] F. Chianucci, et al., "Estimation of Canopy Attributes in Beech Forests Using True Colour Digital Images from A Small Fixed Wing UAV," International Journal of Applied Earth Observation and Geoinformation, vol. 47, pp. 60-68, 2016.

[19] W. Khokthong, et al., "Drone-Based Assessment of Canopy Cover for Analyzing Tree Mortality in an Oil Palm Agroforest," Frontiers in Forests and Global Change, vol. 2, no. 12, 2019.

[20] C. Huang, et al., "A Strategy for Estimating Tree Canopy Density Using Landsat 7 ETM+ and High Resolution Images Over Large Area," Third Int. Conference on Geospatial Information in Agriculture and Forestry, 2001. 
[21] D. A. Umarhadi, et al., "The Comparison of Canopy Density Measurement Using UAV and Hemispherical Photography for Remote Sensing Based Mapping," 4th International Conference on Science and Technology (ICST), pp. 1-5, 2018.

[22] R. D. Dimyati, et al., "A Minimum Cloud Cover Mosaic Image Model of the Operational Land Imager Landsat-8 Multitemporal Data using Tile based," International Journal of Electrical and Computer Engineering (IJECE), vol. 8, no. 1, pp. 360-371, 2018.

[23] M. Palmolina, "Pengelolaan Hutan Rakyat pada Lahan Sempit-Community Forest Management in Narrow Land (in Bahasa)," Prosiding Seminar Nasional Masyarakat Biodiversitas Indonesia, pp. 732-737, 2015.

[24] S. Dhingra and D. Kumar, "A Review of Remotely Sensed Satellite Image Classification," International Journal of Electrical and Computer Engineering (IJECE), vol. 9, no. 3, pp. 1720-1731, 2019.

[25] M. Xu, X Jia, M Pickering., "Automatic Cloud Removal for Landsat 8 OLI Images Using Cirrus Band," IEEE Geoscience and Remote Sensing Symposium, pp. 2511-2514, 2014.

[26] D. A. Umarhadi and P. Danoedoro, "Correcting topographic effect on Landsat-8 images: an evaluation of using different DEMs in Indonesia," Proc. SPIE 11311, Sixth Geoinformation Science Symposium, 2019.

[27] W. Rouse, et al., "Monitoring Vegetation Systems in the Great Plains with ERTS," Proc. Third Earth Resources Technology Satellite-1 Symposium, SP-351, 3010-3017, 1974.

[28] J. Qi, et al., "A Modified Soil Adjusted Vegetation Index," Remote Sensing of Environment, vol. 48, no. 2, pp. 119-126, 1994.

[29] A. Karnieli, et al., "AFRI-Aerosol Free Vegetation Index," Remote Sensing of Environment, vol. 77, no. 1, pp. 10-21, 2001.

[30] R. M. McCoy, "Field Methods in Remote Sensing," The Guilford Press A Division of Guilford Publications, 2005.

[31] H. Zheng, et al., "Evaluation of RGB, Color-Infrared and Multispectral Images Acquired from Unmanned Aerial Systems for the Estimation of Nitrogen Accumulation in Rice," Remote Sensing, vol. 10, no. 6, 2018.

[32] M. Weiss and F. Baret, "Can Eye V6.4.91 User Manual," INRA Science \& Impact, 2017.

[33] D. D. Gupita and S. H. M. B. Santosa, "Soil Erosion and Its Correlation with Vegetation Cover: An Assessment Using Multispectral Imagery and Pixel-Based Geographic Information System in Gesing Sub-Watershed, Central Java, Indonesia," IOP Conf. Series: Earth and Environmental Science, vol. 54, no. 1, 2017.

[34] S. B. Jennings, ND Brown, D Sheil., "Assessing Forest Canopies and Understorey Illumination: Canopy Closure, Canopy Cover and Other Measures," Forestry, vol. 72, no. 1, pp. 59-74, 1999.

[35] M. Kamal, S Phinn, K Johansen, "Characterizing the Spatial Structure of Mangrove Features for Optimizing Image-Based Mangrove Mapping,” Remote Sensing, vol. 6, no. 2, pp. 984-1006, 2014.

\section{BIOGRAPHIES OF AUTHORS}

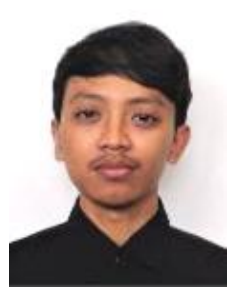

Deha Agus Umarhadi, was born in Yogyakarta. He obtained his B.Sc of Cartography and Remote Sensing in Faculty of Geography, Universitas Gadjah Mada in 2017. He was a research assistant at Center for Remote Sensing and Integrated Surveys (PUSPICS), Faculty of Geography, Universitas Gadjah Mada (2017-2019). Currently, he is a master's student at Graduate School of Environmental Science, Faculty of Environmental Earth Science, Hokkaido University.

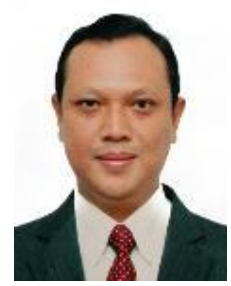

Projo Danoedoro, was born in Yogyakarta. He obtained his B.Sc in the field of Technical Geography/Remote Sensing in Faculty of Geography, Universitas Gadjah Mada in 1989, and M.Sc from the Department of Land Resources and Urban Science (LARUS), Rural Land Ecology Survey Division, ITC in 1993. He obtained his Ph.D in Centre for Remote Sensing and Spatial Information Science (CRSSIS), The University of Queensland (UQ), Australia (2007). He was the Director of Center for Remote Sensing and Integrated Surveys (PUSPICS) (20082017). Currently, he is the Head of Remote Sensing Laboratory, and the Head of Remote Sensing and GIS Graduate Program, Faculty of Geography, Universitas Gadjah Mada. 